\title{
Complex Dynamics and Statistics of 1-D Hamiltonian Lattices: Long Range Interactions and Supratransmission
}

\author{
Anastasios Bountis \\ Department of Mathematics, Nazarbayev University, \\ Kabanbay - Batyr 53, Nur-Sultan, 010000, Republic of Kazakhstan
}

(Received 14 November, 2019)

\begin{abstract}
In this paper, I review a number of results that my co-workers and I have obtained in the field of 1-Dimensional (1D) Hamiltonian lattices. This field has grown in recent years, due to its importance in revealing many phenomena that concern the occurrence of chaotic behavior in conservative physical systems with a high number of degrees of freedom. After the establishment of the Kolomogorov-Arnol'd-Moser (KAM) theory in the 1960s, a wealth of results were obtained about such systems as small perturbations of completely integrable Ndegree-of-freedom Hamiltonians, where ordered motion is dominant in the form of invariant tori. Since the 1980s, however, and particularly in the last two decades, there has been great progress in understanding the properties of Hamiltonian 1D lattices far from the KAM regime, where "weak" and "strong" forms of chaos begin to play an increasingly significant role. It is the purpose of this review to address and highlight some of these advances, in which the author has made several contributions concerning the dynamics and statistics of these lattices.
\end{abstract}

PACS numbers: 05.45.-a

Keywords: N-degree-of-freedom Hamiltonian system, dynamics, statistics

DOI: https://doi.org/10.33581/1561-4085-2020-23-2-133-148

\section{Introduction}

Hamiltonian systems constitute one of the most active fields of research in what we call today Nonlinear Science. They are among the first to which the ideas and methods of Nonlinear Dynamics and Chaos were successfully applied and yielded a wealth of results that revolutionized many areas of Classical and Quantum Mechanics, Astronomy, Plasma Physics, Statistical Mechanics and Solid State Physics. They constitute dynamical systems said to possess $N$ degrees of freedom, whose time evolution occurs in an $n=2 N$ dimensional phase space of position and momentum coordinates $q_{k}(t), p_{k}(t)$ respectively, $k=1,2, \ldots, N$, with $n=2 N$, and whose equations of motion are given by $[1-4]$ :

$$
\frac{d q_{k}}{d t}=\frac{\partial H}{\partial p_{k}}, \frac{d p_{k}}{d t}=-\frac{\partial H}{\partial q_{k}}, k=1,2, \ldots, N
$$

where $H=H\left(p_{1}, p_{2}, \ldots, p_{N}, q_{1}, q_{2}, \ldots, q_{N}\right)$ is the Hamiltonian function. Since we assume in (1) that $H$ does not explicitly depend on time $t, H$ is a first integral of the motion and its value $E$ equals the total energy of the system. Let us now consider an $H$ that can be expanded in power series as a sum of homogeneous polynomials $H_{m}$ of degree $m \geq 2$.

$$
H=\sum_{m, m \geq 2} H_{m}\left(q_{1}, \ldots, q_{N}, p_{1}, \ldots, p_{N}\right)=E,
$$

so that the origin $q_{k}(t)=p_{k}(t)=0, k=$ $1,2, \ldots, N$, is an equilibrium point of the system. Thus, $H=E$ defines a $(2 N-1)$ dimensional manifold called the (constant) energy surface, on which the dynamics evolves. We also assume that linearizing equations (1) about the origin generates a matrix, whose eigenvalues arise in conjugate imaginary pairs, $\pm i \omega_{k}, k=1, \ldots, N$, providing the $N$ frequencies of the normal mode oscillations of the linear problem. Then, according to a famous theorem by Lyapunov [5], if there are no rationals among the ratios $\omega_{j} / \omega_{k}$, for any $j, k=1,2, \ldots, N$ where $j \neq k$, then the linear modes persist as periodic solutions of the nonlinear problem, with frequencies close to those of the linear modes and will be called simple periodic orbits (SPOs) as all particles return to their original position after one oscillation.

Consider one simple example of such a system, with $N=2$ degrees of freedom, described 
by the Hamiltonian

$H=H_{0}+\epsilon H_{1}=\frac{1}{2} p_{x}^{2}+\frac{1}{2} p_{y}^{2}+\frac{x^{4}}{2}+\frac{y^{4}}{2}+\epsilon x^{2} y^{2}=E$

where we have set $p_{x}=p_{1}, p_{y}=p_{2}$ and $q_{1}=x$, $q_{2}=y$ to denote the two degrees of freedom of the problem. To study the dynamics, we introduce the so-called Poincaré Surface of Section (PSS) defined in the $x, p_{x}$ plane, for different initial conditions, by points where $y=0$ and $p_{y}>0$, at a given value of $E$, see Fig. 1. In fact, in Fig. 1(a) we set $\epsilon=0$ in eq. (3) and note that the PSS is filled with closed orbits, which are either periodic or quasiperiodic, depending on whether the ratio of the frequencies of the two oscillators is rational or irrational. Since the motion is governed by two independent integrals of the motion, no chaos is expected in this case as all orbits are regular [1-4].

We now impose coupling between the oscillators, letting $\epsilon=0.02$, and consider the PSS obtained for the same energy value $E$ as in Fig. 1(a). What we find now in Fig. 1(b), as expected from the theory, is that, while many regular quasiperiodic solutions still exist, the periodic curves of Fig. 1(a) have split into chains of stable orbits (represented by islands) interlaced by chains of unstable orbits (shown as saddle points). At this scale, no chaotic motions are visible. Magnifying, however, the figure near one of its saddle points, reveals in the inset of Fig. 1(b) a highly complex type of dynamics, whose most distinctive feature is the presence of thin layers of chaos, which in fact extend around the islands and join with the corresponding chaotic regions of other saddles belonging to the same family.

Interestingly, these chaotic domains grow in size significantly on PSS that correspond to higher values of $\epsilon=0.2$, see Fig. 1(c). Several important questions arise from this simple example, which we will venture to address in the present paper:

- What happens at higher values of $\epsilon$ or, equivalently, higher values of the energy $E$ in such examples? Will there always exist "wide" and "thin" chaotic regions at different scales and if so, how can we characterize their dynamics? Can we say that within the thinner such layers a "weaker" form of chaos occurs, compared to the wider domains where the chaos is "stronger"?
- If indeed such distinction between "strong" and "weak" chaos can be made, how can we differentiate between them studying their dynamical and statistical properties?

- What about those stable low period orbits lying at the center of the largest islands of regular motion shown in Fig. 1(b,c)? Will they continue to be stable for larger values of $\epsilon$ (or $E$ ), and if they do destabilize, what happens immediately after they become unstable? Will we find "strong" or "weak" chaos in their vicinity?

- Last and most important: How do all these phenomena manifest themselves in the case of many degrees of freedom where $N$ is allowed to grow indefinitely?

In Section 2, I will consider all these questions on the fundamental paradigm of a $N-$ degree of freedom 1-D Hamiltonian called the Fermi-Pasta-Ulam-Tsingou model, widely known as the $\beta$-FPU system [6], described by the Hamiltonian

$$
\begin{array}{r}
H=\frac{1}{2} \sum_{j=1}^{N} p_{j}^{2}+\sum_{j=1}^{N} \frac{1}{2}\left(x_{j+1}-x_{j}\right)^{2} \\
+\frac{\beta}{4} \sum_{j=1}^{N}\left(x_{j+1}-x_{j}\right)^{4}=E
\end{array}
$$

where $x_{j}$ are the displacements of the particles from their equilibrium positions and $p_{j}=d x_{j} / d t$ are the momenta, $\beta$ is a positive real constant and $E$ is the total energy. Let us now ask: What would be the simplest periodic solutions of this systems, lying at the center of large islands, similar to those we found in the above $N=2$ example?

In an early work, Chris Antonopoulos and I [7] observed that the FPU system possesses some examples of what we called SPOs, which exist for all energies due to translational symmetries of the 1-D lattice, and are obtained in terms of a single periodic function $\hat{x}(t)$ as follows:

- the out of phase mode (OPM)

$$
\hat{x}_{j}(t)=-\hat{x}_{j+1}(t)=\hat{x}(t), j=1,2, \ldots, N
$$

where $N$ is even, under periodic boundary conditions, i.e. $x_{1}(t)=x_{N+1}(t)$, and 
(a)

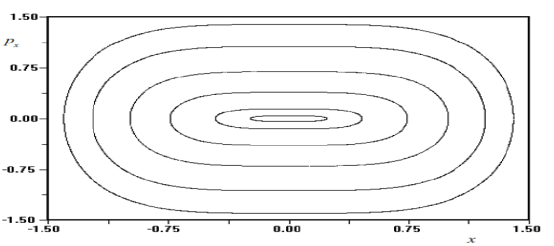

(b)

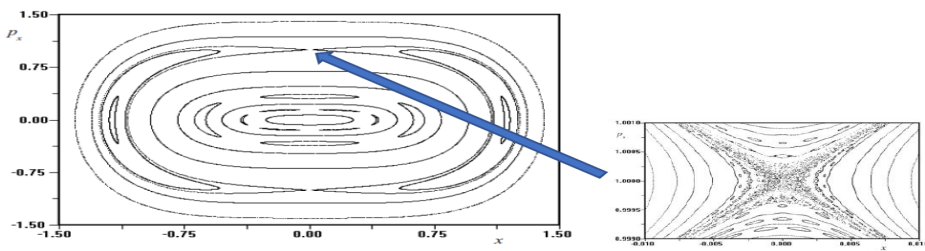

(c)

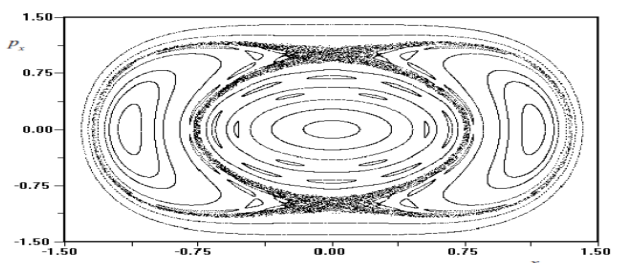

FIG. 1. (color online) (a) Poincaré Surface of Section (PSS) of the solutions in the $x, p_{x}$ plane, defined by $y=0$ and $p_{y}>0$ of the uncoupled oscillators (3) with $\epsilon=0$. (b) Setting $\epsilon=0.02$ we observe that alternating stable (islands) and unstable (saddle points) periodic orbits have appeared, with no chaos visible at that scale. However, magnifying the region near one of the saddles in the inset, we see that "thin" chaotic regions emerge about these saddle points. (c) Increasing the coupling parameter to $\epsilon=0.2$ the chaotic regions grow significantly and "wide" chaotic domains emerge in the figure.

FPU N=4 OPM with fixed boundary conditions
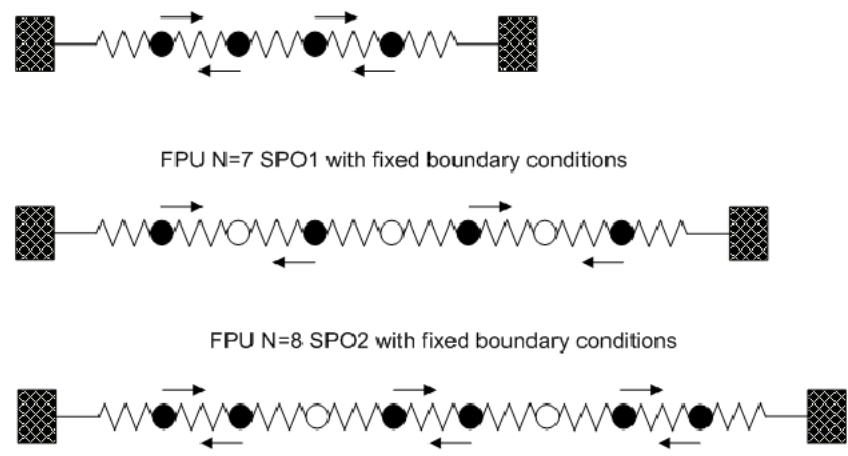

FIG. 2. Examples of SPOs that we have called the Out of Phase Mode (above), the SPO1 (middle) and the SPO2 (below).

- the SPO1 mode, where for every 2 particles one is stationary while those on its either side move out of phase,

- the SPO2 mode, where for every 3 particles one is stationary and the two on either side move out of phase with respect to each other. Both SPO1 and SPO2 have fixed boundary conditions, i.e. $x_{0}(t)=$ $x_{N+1}(t)=0, p_{0}(t)=p_{N+1}(t)=0$.

One can prove the existence of SPOs for the FPU as continuations of the linear normal modes of the system, whose energies and frequencies are given by $[7,8]$ :

$$
\begin{array}{r}
E_{q}=\frac{1}{2}\left(P_{j}^{2}+\omega_{q}^{2} Q_{q}^{2}\right), \quad \omega_{q}=2 \sin \left(\frac{\pi q}{2(N+1)}\right), \\
q=1,2, \ldots, N
\end{array}
$$

applying Lyapunov's Theorem mentioned above, noting that the ratios of any two of the normal mode frequencies in (6) are irrational, while $P_{q}, Q_{q}$ are the corresponding normal mode coordinates [2]. SPO1 and SPO2 orbits, for example, are two such nonlinear normal modes 


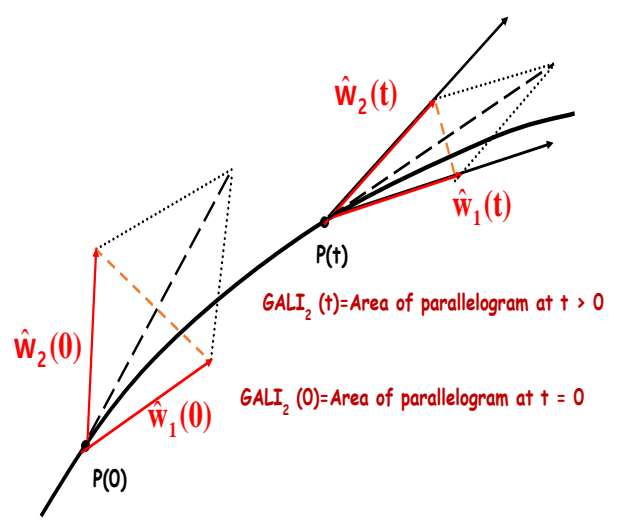

FIG. 3. (color online) Schematic drawing of how the GALI index is defined in terms of two unit deviation vectors (GALI2) in the case of a chaotic orbit: $\hat{\mathbf{w}}_{1}(0)$ and $\hat{\mathbf{w}}_{2}(0)$ are initially chosen in two arbitrary linearly independent directions off the chaotic orbit at the point $P(0)$. After some time $t>0$, they become $\hat{\mathbf{w}}_{1}(t)$ and $\hat{\mathbf{w}}_{2}(t)$ and are closer to the direction of the orbit, denoted by a dashed line tending to align with the MLE. As a result the area of the parallelogram they form becomes smaller and tends to zero as $t$ grows.

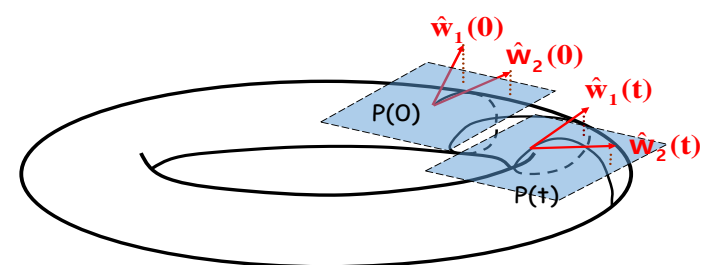

FIG. 4. (color online) Schematic drawing of how the GALI index is defined in terms of two unit deviation vectors (GALI2) in the case of a regular orbit lying on a 2 -D torus: $\hat{\mathbf{w}}_{1}(0)$ and $\hat{\mathbf{w}}_{2}(0)$ are initially chosen in two arbitrary linearly independent directions off the torus at the point $P(0)$. After some time $t>0$, they become $\hat{\mathbf{w}}_{1}(t)$ and $\hat{\mathbf{w}}_{2}(t)$, are closer to the torus and tend to fall on its tangent space. As they remain linearly independent, the area of the parallelogram they define tends to a constant value.

identified by the indices $q=\frac{N+1}{2}$ and $q=\frac{2(N+1)}{3}$, respectively, in equations (6).

In $[7,8]$, the linear stability analysis of the above orbits was presented, which due to their simplicity can be carried out for as high $N$ as we desire. Thus, we discovered that, while these orbits are stable at low energies, as $E>$ 0 grows, they first become unstable at critical values $E_{c}(N)$ which tend to zero as $N \rightarrow \infty$ following specific power laws. We thus ask what happens near some of them when they do become unstable. How wide is the chaotic domain in their vicinity? Does the motion evolve in thin layers of "weak" chaos or does it immediately spread out in wide,"strongly" chaotic domains?

Next, in Section 2, I recall two different approaches that my co-workers and I have employed in such investigations: The first makes use of the Maximum Lyapunov Exponent (MLE) [22] and its extension, the Generalized Alignment Index (GALI), to distinguish between dynamical properties of order and chaos $[9,10]$. The second appeals to probability density functions (pdfs) of position and momentum coordinates, which reveal the statistical properties of different chaotic regimes and are thus able to distinguish between "weak" and "strong" chaos [11]. Both of these approaches will be applied here to the above FPU model to study the dynamics and statistics of its SPO1 solution, in the case of $N=5$ particles (or degrees of freedom).

Note now that 1-D Hamiltonian lattices like (4) involve only interactions between nearest neighbors, which are of the shortest possible range, since each particle is affected only by its two closest neighbors. An interesting question, therefore, taken up by Helen Christodoulidi, Constantino Tsallis, Lambros Drossos and myself is what happens to the dynamics and statistics of the model in the case of "long range interactions" (LRI) where each particle is allowed to interact with all others through a coefficient of the form $1 / r^{\alpha}$ where $r$ denotes the inter-particle distance and $0 \leq \alpha \leq \infty[13,14]$. We will pick up this topic in Section 3, where we will show that LRI appear to have a globally "stabilizing" effect" on the system! In other words, we will discover that as the particles tend to interact equally with all others, the system becomes dynamically more ordered and statistically more "weakly chaotic"!

In Section 4, I will revisit more recent results obtained by Jorge Macias Diaz, Helen Christodoulidi and myself, concerning what happens to the FPU 1-D lattice under LRI, when the first particle at the left obeys the moving boundary condition $x_{1}(t)=A \sin \Omega t$. Other researchers had already discovered, on a number of nearest-neighbor 1-D Hamiltonians, the fascinating phenomenon of supratransmission where, for a well-defined range of $\Omega$ values, a precise amplitude $A_{c}$ exists such that for $A>$ $A_{c}$ a sudden surge of energy propagates in the lattice. We first showed that supratransmission 
also occurs in the FPU model under LRI, albeit at considerably higher $A_{c}$ values than the nearest neighbor case! We then went on to study this phenomenon on LRI lattices of the Sine Gordon type in the presence of on site potentials, which allow for localized oscillations called breathers $[15,16]$.

The paper ends with a section on Future Outlook where I discuss work in progress on Hamiltonian 1-D lattices with non analytic potentials, arising in problems of complex materials and nonlinear elasticity. Performing similar studies of their dynamical stability and chaotic behavior based on their SPOs, preliminary results suggest that they possess strikingly different properties than what we have found for Hamiltonians of the FPU type.

\section{Dynamical and statistical analysis of the $\beta-$ FPU model}

\subsection{The dynamical criteria of Lyapunov exponents and GALI indicators}

The Lyapunov characteristic exponents (LCEs) of a Hamiltonian dynamical system

$L_{i}, \quad i=1, \ldots, 2 N, L_{1} \equiv L_{\max }>L_{2}>\ldots>L_{2 N}$

measure the rate of exponential divergence of initially nearby orbits in the phase space of the dynamical system. The LCEs come in pairs of opposite sign, and two are equal to zero corresponding to deviations along the orbit. If the largest one, $L_{1} \equiv L_{\max }>0$ (MLE), the orbit is chaotic, i.e. almost all nearby orbits diverge exponentially in time, while if $L_{\max }=0$ the orbit is stable. The numerical algorithm used most often for the computation of all LCEs was proposed in [17]. The $L_{i}$ for a given orbit $\mathbf{x}(t)$ expresses the limit for $t \rightarrow \infty$ of a quantity of the form

$$
K_{t}^{i}=\frac{1}{t} \ln \frac{\left\|\mathbf{w}_{i}(t)\right\|}{\left\|\mathbf{w}_{i}(0)\right\|}, L_{i}=\lim _{t \rightarrow \infty} K_{t}^{i}
$$

where $\mathbf{w}_{i}(0)$ and $\mathbf{w}_{i}(t), \quad i=1, \ldots, 2 N-$ 1 are infinitesimal deviation vectors from the given orbit $\mathbf{x}(t)$ that are orthogonal to the vector tangent to the orbit. Note now that, in the computation of LCEs, one considers only one deviation from the orbit each time. We decided, therefore, in $[9,10]$ to find out what happens if we consider more deviations and defined the index $\mathrm{GALI}_{k}$ as the volume of the $k$-parallelepiped having as edges the $k$ unit deviation vectors $\hat{\mathbf{w}}_{i}(t)=\mathbf{w}_{i}(t) /\left\|\mathbf{w}_{i}(t)\right\|, i=$ $1,2, \ldots, k$, determined through the wedge product of these vectors as

$$
\operatorname{GALI}_{k}(t)=\left\|\hat{\mathbf{w}}_{1}(t) \wedge \hat{\mathbf{w}}_{2}(t) \wedge \cdots \wedge \hat{\mathbf{w}}_{k}(t)\right\|
$$

where $\|\cdot\|$ denotes the usual norm. From this definition it is evident that if at least two of the deviation vectors become linearly dependent, the wedge product in (9) becomes zero and the $\mathrm{GALI}_{k}$ vanishes. Geometrically, of course, $G A L I_{k}$ represents the volume of the $k$-"parallelepiped" formed by these vectors and thus, if at least 2 of the deviations are linearly dependent, the $G A L I_{k}$ vanishes.

Let us see how all this works for the case of $k=2$ such deviations. In Fig. 3, we see schematically that $G A L I_{2}$ represents the area of the parallelogram formed by the deviation vectors, and goes to zero as $t>0$ increases, since these vectors tend to align with the most unstable direction identified by the MLE. We thus conclude that the orbit under study in this case is chaotic. On the other hand, if the orbit were regular and evolved on a 2-D torus (e.g. in the case of a Hamiltonian system of $N=2$ degrees of freedom), see Fig. 4, the two deviation vectors would tend to fall on the tangent space of the torus and hence the area of the parallelogram they define would never vanish. This means that $G A L I_{2} \approx$ constant and the orbit would be termed regular. Writing (9) as a single determinant, expanding it in a sum of subdeterminants of decreasing magnitude, as $t \rightarrow \infty$, and keeping the largest one, we studied in $[9,10]$ the asymptotic behavior of the $G A L I_{k}$, and obtained the following analytical result: In the case of a chaotic orbit, all deviation vectors tend to become linearly dependent, aligning in the direction defined by the MLE, and all GALI $k$ tend to zero exponentially following the law:

$$
G A L I_{k}(t) \approx e^{-\left[\left(L_{1}-L_{2}\right)+\left(L_{1}-L_{3}\right)+\cdots+\left(L_{1}-L_{k}\right)\right] t}
$$

where $L_{1}, \ldots, L_{k}$ are the $k$ largest LCEs. On the other hand, if the orbit under study is regular, matters are more subtle: All deviation vectors tend to fall on the $s$-dimensional tangent space 
of the torus on which the motion lies. Thus, if we start with $k \leq s$ general deviation vectors they will remain linearly independent on the $s$ dimensional tangent space of the torus, since there is no reason for them to become aligned. As a consequence, $\mathrm{GALI}_{k}$ remains practically constant and different from zero for $k \leq s$. On the other hand, $\mathrm{GALI}_{k}$ tends to zero for $k>s$, since some deviation vectors will eventually become linearly dependent. In particular, the generic behavior of $\mathrm{GALI}_{k}$ for quasiperiodic orbits lying on $s$ dimensional tori is given by $[9,10]$ :

$$
\operatorname{GALI}_{k}(t) \propto\left\{\begin{array}{ll}
\text { constant } & \text { if } 2 \leq k \leq s \\
\frac{1}{t^{k-s}} & \text { if } s<k \leq 2 N-s \\
\frac{1}{t^{2(k-N)}} & \text { if } 2 N-s<k \leq 2 N
\end{array} .\right.
$$

At this point we make a crucial observation: Note that the above criterion allows us to determine the dimension $s$ of any torus under study! Starting with many deviation vectors, i.e. $k>s$ and gradually decreasing $k$, the torus dimension is determined by the particular value of $k=s$ at which GALIs cease to tend to zero and become constant. To this day, I do not know of a better way to determine the dimension of a torus, especially in spaces of high dimensionality.

Let us apply these criteria to regular orbits of two specific examples of 2 and 3 degree of freedom Hamiltonians respectively, as shown in Fig. 5. In the case of Fig. 5(a) the torus is two dimensional so $G A L I_{2}$ is nearly constant, while all higher order GALIs tend to zero following power laws that agree with the analytical result shown in (11). A similar situation is depicted in Fig. 5(b), only here $G A L I_{2}$ and $G A L I_{3}$ are nearly constant, since the torus is 3-dimensional.

\subsection{A statistical criterion based on the Central Limit Theorem}

As is well-known, multi-particle systems belong to different universality classes, according to their statistical properties at equilibrium. In the most widely studied class, if the system can be at any one of $i=1,2, \ldots, W$ states with probability $p_{i}$, its entropy is given by the celebrated Boltzmann-Gibbs (BG) formula

$$
S_{B G}=-k \sum_{i=1}^{W} p_{i} \ln p_{i}
$$

where $k$ is Boltzmann's constant, under the constraint

$$
\sum_{i=1}^{W} p_{i}=1
$$

As is well-known, the BG entropy is additive in the sense that, for any two independent systems $A$ and $B$, the entropy of their sum is the sum of the individual entropies, i.e. $S_{B G}(A+B)=$ $S_{B G}(A)+S_{B G}(B)$. It is also extensive, as it grows linearly with $N$, as $N \rightarrow \infty$. These properties are associated with the fact that different parts of BG systems are highly uncorrelated and their dynamics is statistically independent in phase space.

There is, however, an abundance of physical systems characterized by strong correlations, for which the assumptions of extensivity and additivity are not generally valid [18]. In fact, Hamiltonian systems provide a wealth of examples governed by such correlated statistics, especially near the boundaries of islands of regular motion where orbits "stick" for very long times and the dynamics becomes very weakly chaotic. It is for this kind of systems that Tsallis proposed the entropy formula

$$
S_{q}=k \frac{1-\sum_{i=1}^{W} p_{i}^{q}}{q-1} \text { with } \sum_{i=1}^{W} p_{i}=1
$$

that depends on an index $q$, for a set of $W$ states with probabilities $p_{i} i=1, \ldots, W$, obeying the constraint (13). The $S_{q}$ entropy is not additive, since $S_{q}(A+B)=S_{q}(A)+S_{q}(B)+k(1-$ q) $S_{q}(A) S_{q}(B)$ and generally not extensive. The pdf replacing the Gaussian in this case is the $q$ Gaussian distribution

$$
P(s)=a \exp _{q}\left(-\beta s^{2}\right) \equiv a\left[1-(1-q) \beta s^{2}\right]^{\frac{1}{1-q}}
$$

obtained as an extremum of the Tsallis entropy (14), under appropriate constraints [18]. The $q$ index satisfies $1<q<3$ to make (15) 

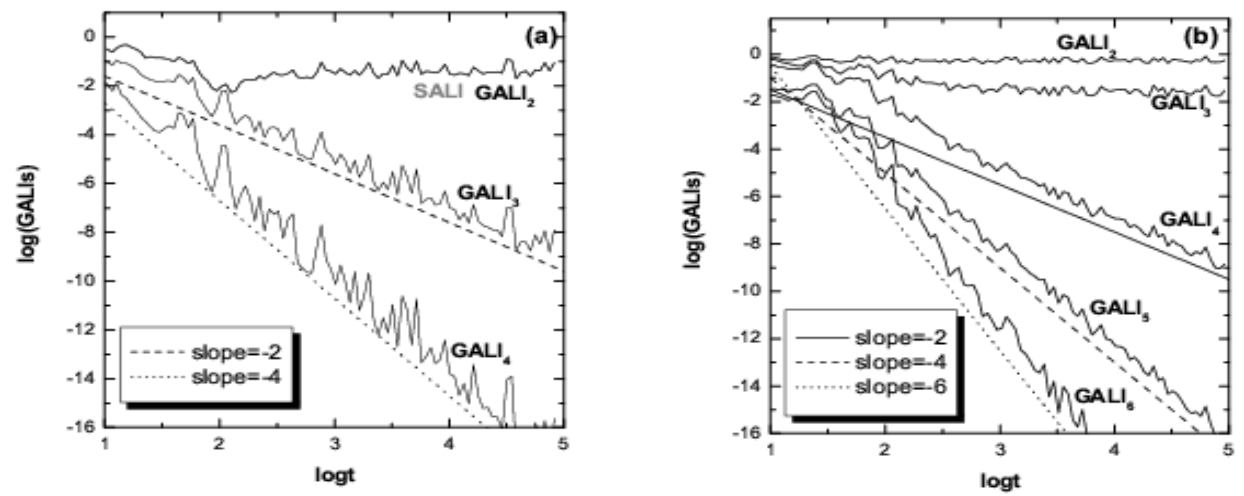

FIG. 5. Computation of $G A L I_{k}(t)$ as time evolves, for a regular orbit belonging to: (a) a Hamiltonian system of 2 degrees of freedom and (b) a 3 degree of freedom Hamiltonian. Note that in (a) only $G A L I_{2}$ is nearly constant, since the torus has dimension 2, while in (b) both $G A L I_{2}$ and $G A L I_{3}$ are nearly constant since the torus is 3-dimensional. All other GALIs tend to zero with power laws that are in very good agreement with our asymptotic result (11) (after $[9,10])$.

normalizable, $\beta$ is an arbitrary parameter and $a$ a normalization constant. Note that in the limit $q \rightarrow 1(15)$ tends to the Gaussian distribution, i.e. $\exp _{q}\left(-\beta x^{2}\right) \rightarrow \exp \left(-\beta x^{2}\right)$ of $\mathrm{BG}$ statistical mechanics.

Our approach, concerning $N$ degree of freedom Hamiltonians, is in the spirit of the wellknown Central Limit Theorem and is described in detail in $[4,19]$. In particular, we solve Hamilton's equations of motion for a large set of initial conditions and obtain pdfs of suitably rescaled sums of the system's positions or momenta, centered about their mean value and rescaled by their standard deviation. What we find is that, in cases of "strong" chaos the pdfs converge to the BG exponential law, as time increases, while in "weakly" chaotic situations they tend to follow a $q$-Gaussian distribution. Let us see how all this applies to the case of an SPO1 orbit of our FPU Hamiltonian (4) with $N=5$ at an energy value where the orbit has just turned unstable, see Fig. 6. Plotting its intersections with the plane of position and momentum coordinates of the first particle, $q_{1}, p_{1}$, at times when the third particle has $q_{3}=0$, we observe in Fig. 6 , starting very close to the SPO1 orbit, that successive intersections form a "figure 8" type of chaotic domain in phase space. This thin layer of chaos expands a little when we move further away from the SPO1 orbit, while moving even further away the solutions eventually abandon the "figure 8" and explore a much larger chaotic component of phase space. Let us see now what happens when we compute pdfs of position coordinates for the three cases of chaotic domains obtained for the three initial conditions of Fig. 6 . As we show in Fig. 7, as long as our orbit remains "trapped" within the "figure 8" region of weak chaos (what is often referred as "sticking phenomenon") its statistical distribution is well represented by a q-Gaussian and hence obeys Tsallis thermostatistics, while as it moves further away to strong chaos its pdf tends towards a pure Gaussian associated with BG statistical mechanics.

\section{The effect of long range interactions}

Note that the results we have described so far, connecting regions of weak and strong chaos in the $\beta$-FPU chain to Tsallis and BG thermostatistics respectively, originally came as a surprise to many researchers. The reason is that the FPU chain is a system that involves only nearest-neighbor particle interactions, which are of the shortest range type. How then can such a system exhibit Tsallis thermostatistics, which is expected to arise only in systems involving long range interactions (LRI)?

The answer to this question is rather evident: LRI does not only refer to a global property characterizing a given system; it can also be a local property governing the dynamics in localized domains of phase space. Thus, even though the $\beta-\mathrm{FPU}$ lattice with nearest neighbor interactions is not a globally long range system, it possesses LRI locally, e.g. in cases where the orbit displays the phenomenon of "stickiness", in thin chaotic 
layers and very close to the boundaries of islands of regular motion.

\subsection{LRI only on the nonlinear} interactions

To study, therefore, what happens in a system which has global LRI, we chose initially to take a $\beta-F P U$ 1D lattice described by the Hamiltonian [11]

$$
\begin{array}{r}
\mathcal{H}=\frac{1}{2} \sum_{n=1}^{N} p_{n}^{2}+\frac{1}{2} \sum_{n=0}^{N}\left(x_{n+1}-x_{n}\right)^{2}+\frac{b}{4 \widetilde{N}} \\
\times \sum_{n=0}^{N} \sum_{m=n+1}^{N+1} \frac{\left(x_{n}-x_{m}\right)^{4}}{|n-m|^{\alpha}}=U(N), b>0 ; \alpha \geq 0
\end{array}
$$

with fixed boundary conditions, i.e. $x_{0}=x_{N+1}=$ $p_{0}=p_{N+1}=0$. Note here that LRI is applied only to the quartic part of the potential, scaled by a factor $N^{-1}$, which varies with $N$ as follows:

$$
\begin{array}{r}
\tilde{N}(N, \alpha) \equiv \frac{1}{N} \sum_{i=0}^{N} \sum_{j=i+1}^{N+1} \frac{1}{(j-i)^{\alpha}} \\
=\frac{1}{N} \sum_{i=0}^{N} \frac{N+1-i}{(i+1)^{\alpha}}
\end{array}
$$

and whose role is to make the total kinetic and potential energy extensive (i.e. proportional to $N$ ) for all values of $\alpha$. The two limits (i) $\alpha \rightarrow 0$ and (ii) $\alpha \rightarrow \infty$ correspond to the extremal cases where (i) each particle interacts equally with all others, and (ii) only interactions with nearest neighbors apply, recovering exactly the Hamiltonian of the FPU- $\beta$ model. We began our study with a systematic investigation of the largest Lyapunov exponent $\lambda_{\max }$ characterizing the ergodicity of the dynamics for different values of $\alpha, N$ and specific energies $\epsilon=U(N) / N$. In Fig. 8 we thus plotted the $\lambda_{\max }$ versus the system size $N$ for different $\alpha$ values, ranging from 0 to 10 . The critical value $\alpha=1$, similar to what was found in [12], clearly distinguishes between the following two distinct regimes:

i) For $\alpha \geq 1$ the Lyapunov exponent $\lambda_{\max }$ tends to stabilize at a finite and positive value as $N$ increases. ii) For $\alpha<1$ the largest Lyapunov exponents are observed to decrease with system size as $N^{-\kappa(\alpha)}$ where the dependence of the exponent $\kappa(\alpha)$ on $\alpha$ is shown in the inserted panel of Fig.8. We therefore expect that the system with short-range interactions tends to a BG type of equilibrium in the thermodynamic limit, characterized by "strong" chaos. On the other hand, we observe that the case of long range interactions is much more "weakly" chaotic.

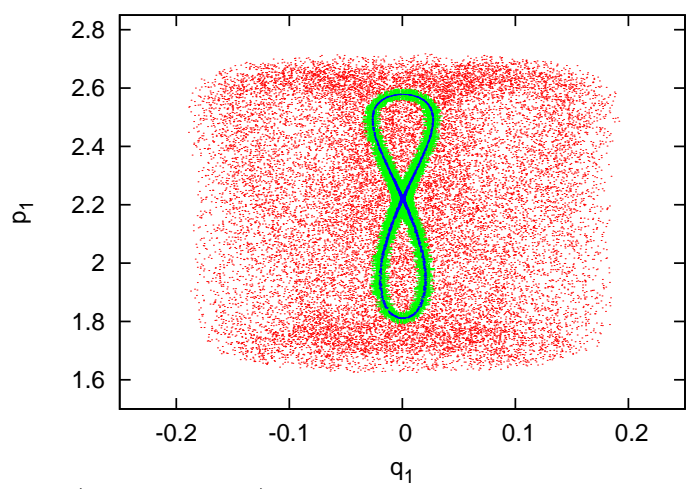

FIG. 6. (color online) A thin "figure 8" chaotic region is observed in the $\left(q_{1}, p_{1}\right)$ plane computed when $q_{3}=$ 0 for an initial condition starting very close to the unstable SPO1 mode passing by the saddle point at $q_{3}=0$ and $p_{3}>0$. A slightly more extended "figure 8" appears for an initial condition further away, while a much wider chaotic region arises for a more distant initial condition. In all cases, we integrate up to $t_{\mathrm{f}}=$ $10^{5}$ with $E=7.4$, and $\beta=1.04$ (after [19]).

Fig. 9 displays the momentum distributions for $\alpha=0.7$ and 1.4 for $N=8192$. In the strong LRI case of $\alpha<1$, the histograms on the left, after sufficiently long times, are well fitted by a $q$-Gaussian pdf with $q=1.249$. However, on the right histogram of Fig. 9 where $\alpha>1$, the distribution follows a pure Gaussian pdf $(q \rightarrow 1$ in (15)) with $\beta=0.043$. The $q$-dependence on $\alpha$ is shown in Fig. 10 where the transition from $q-$ statistics to BG-statistics is evident as $\alpha$ exceeds 1. Starting around $q \simeq 1.32, q$ reaches 1 at $\alpha=1.4$ for $N=16384$ particles calculated during the time interval $\left[5 \cdot 10^{5}, 9 \cdot 10^{5}\right]$. The data of Fig. 10 is averaged over several realizations.

What is most important about these results is that they allow us to draw a "universal phase diagram" in a suitably scaled plane plotted in Fig.11, which displays the crossover between two regimes separated by a straight line in the $1 / N$ vs. 

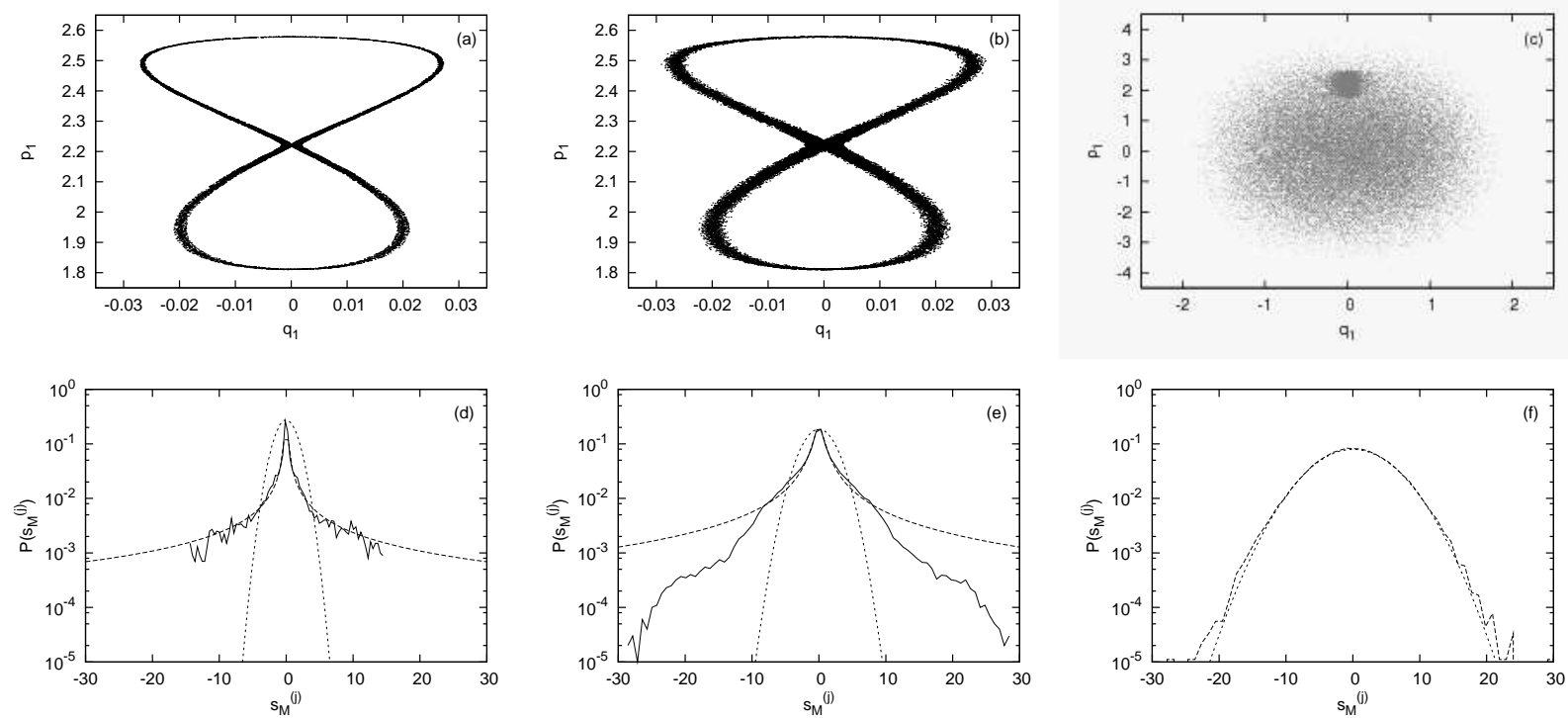

FIG. 7. (a) The $\left(q_{1}, p_{1}\right)$ surface of section of an orbit integrated up to $t_{\mathrm{f}}=10^{5}$ and starting close to the unstable SPO1 orbit of Fig. 6. (b) and (c) are same as (a) but for $t_{\mathrm{f}}=10^{7}$ and $t_{\mathrm{f}}=10^{8}$ respectively. (d)-(f) Plots in linear-log scale of numerical (solid curve), of $q$-Gaussian (dashed curve) and Gaussian (dotted curve) for the initial conditions of (a)-(c) respectively. On the vertical axis of (c), (d) and (e) $s_{M}^{(j)}$ represents averaged sums of position coordinates (after [19]).

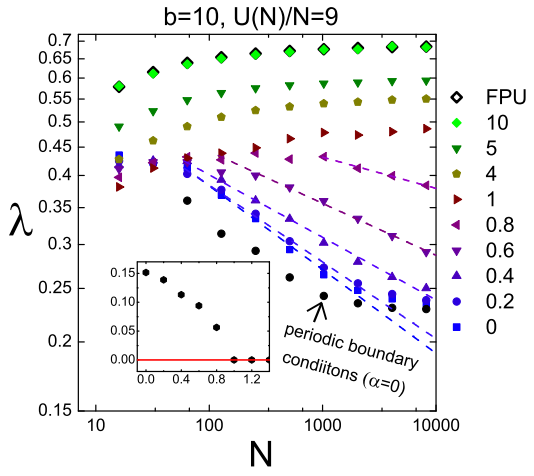

FIG. 8. (color online) Maximal Lyapunov exponent for increasing $N$ and various values of $\alpha$ calculated at $t=10^{6}$, when $U(N) / N=9$, for fixed boundary conditions. Initial conditions are $x_{j}(0)=0$, while $p_{j}(0)$ are chosen randomly from a uniform interval (after [11]).

$b^{\delta} / t_{c}^{\gamma}$ plane. Each point in the graph corresponds to a value of $t=t_{c}$ which represents the maximum time that $q$ remains constant, after which it tends to the $\mathrm{BG}$ value $q=1$.

As a final remark, we point out the nonuniformity of the $(N, t) \rightarrow(\infty, \infty)$ limit implied by the diagram of Fig.11. Clearly, in the $\lim _{N \rightarrow \infty} \lim _{t \rightarrow \infty}$ ordering it is the $q=$
1 BG behavior that prevails, while in the $\lim _{t \rightarrow \infty} \lim _{N \rightarrow \infty}$ ordering it is the Tsallis $q>1$ statistics that becomes dominant.

3.2. LRI on the nonlinear as well as linear interactions

Continuing this line of research in [13], we studied the Fermi-Pasta-Ulam (FPU) $\beta$-model with LRI in both the quadratic and quartic terms in the potential, introducing two independent exponents $\alpha_{1}$ and $\alpha_{2}$ respectively. Our results demonstrate that weak chaos, in the sense of decreasing Lyapunov exponents, and $q$-Gaussian probability density functions (pdfs) of sums of the momenta, occurs only when LRI are included in the quartic part. On the other hand, when LRI are imposed only on the quadratic part, strong chaos and purely Gaussian pdfs are always obtained.

To see all this, let us modify the classical form of the FPU $\beta$-model introducing particle interactions that decay with distance as $1 / r^{\alpha_{1}}$ and $1 / r^{\alpha_{2}}$ respectively. Thus, the modified Hamiltonian function that describes our system 

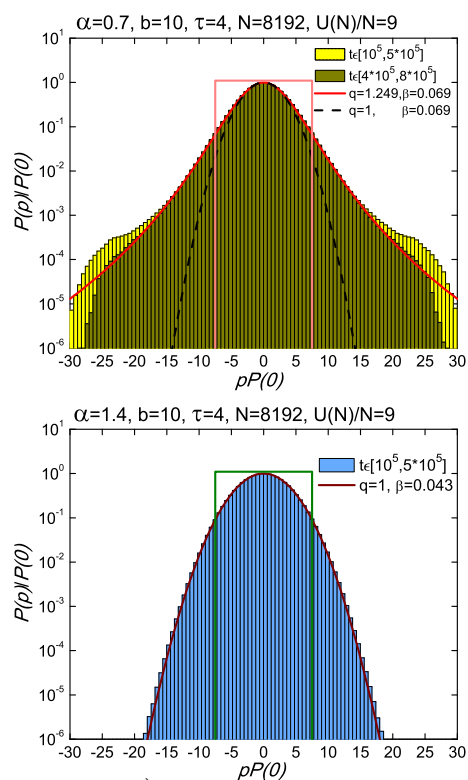

FIG. 9. (color online) Momentum distributions for the system with $N=8192$ and: (up) $\alpha=0.7$ in two time intervals, (down) $\alpha=1.4$. Initial conditions are $x_{j}(0)=0$, while $p_{j}(0)$ are chosen randomly from a uniform interval (after [11]).

has the form [13]

$$
\begin{aligned}
\mathcal{H}_{L R I}=\frac{1}{2} \sum_{n=1}^{N} p_{n}^{2} & +\frac{a}{2 \widetilde{N}_{1}} \sum_{n=0}^{N} \sum_{m=n+1}^{N+1} \frac{\left(x_{n}-x_{m}\right)^{2}}{(m-n)^{\alpha_{1}}} \\
& +\frac{b}{4 \widetilde{N}_{2}} \sum_{n=0}^{N} \sum_{m=n+1}^{N+1} \frac{\left(x_{n}-x_{m}\right)^{4}}{(m-n)^{\alpha_{2}}}
\end{aligned}
$$

where $a$ and $b$ are positive constants. The rescaling factors $\widetilde{N}_{i}, i=1,2$ in (16) are given by the expression

$\widetilde{N}_{i}\left(N, \alpha_{i}\right) \equiv \frac{1}{N} \sum_{n=0}^{N} \sum_{m=n+1}^{N+1} \frac{1}{(m-n)^{\alpha_{i}}}, i=1,2(19)$

and are necessary for making the Hamiltonian extensive. Indeed, without this factor the corresponding sums in (16) would increase as $O\left(N^{2}\right)$ in the thermodynamic limit. Notice that $\widetilde{N}_{i} \simeq 1$ in the limit $\alpha_{i} \rightarrow \infty$, and thus for large $N$ Hamiltonian (18) reduces to Hamiltonian (16). In the four panels of Fig. 12 typical momentum histograms are shown, which correspond to the four representative cases we studied, as

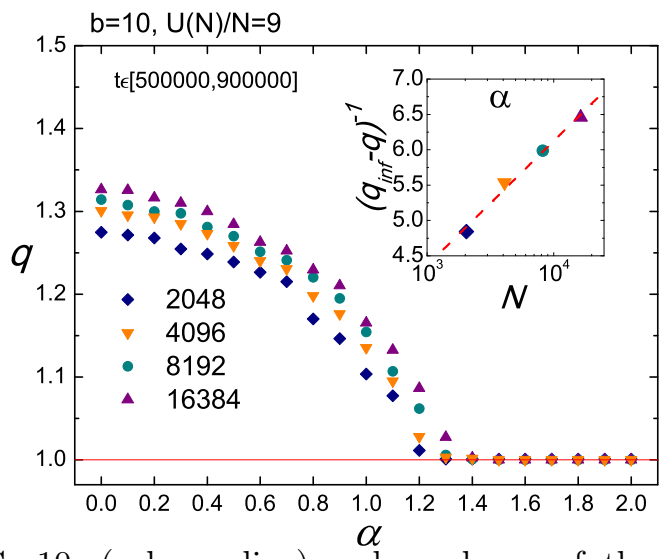

FIG. 10. (color online) $\alpha$-dependence of the index $q$ for $b=10$ and $U(N) / N=9$ averaged over 4 independent realizations when $N$ is 2048, 4096, 8192 and 2 realizations for $N=16384$, all taken in the time interval $t \in\left[5 \cdot 10^{5}, 9 \cdot 10^{5}\right]$. Inserted panel shows $\left(q_{\infty}-q\right)^{-1}$ versus $N$, for the data of the main figure with $\alpha=0 . q_{\infty}$ has a value estimated around 1.48, and is the intercept of the linear dependence of $q$ on $1 / \log N$. The fitting line shown is $\left(q_{\infty}-q\right)^{-1}=$ $1.76 \log N-0.9$ (after [11]).

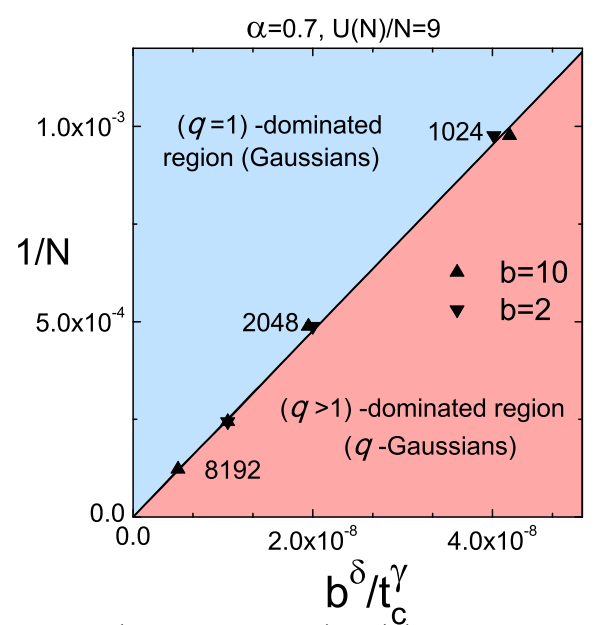

FIG. 11. (color online) (a) "Phase diagram" depicting the crossover between Gaussian and $q-$ Gaussian thermostatistics for the system sizes $N=$ 1024, 2048, 4096,8192 at specific energy $\epsilon=9$ (after [11])

different combinations of short and long-range interactions. More specifically, in panels (a) and (c) a classical Gaussian shape is observed, either under purely short-range interactions or when LRI apply only to the quadratic part, by setting $\alpha_{1}=0.7$ and $\alpha_{2} \rightarrow \infty$ in the Hamiltonian (18). Instead in the panels (b) and (d) a clear $q^{-}$ 
Gaussian shape emerges when long-range applies to the quartic interactions, independently of the type of interactions in the quadratic part, i.e. for $\alpha_{1} \rightarrow \infty, \alpha_{2}=0.7$ and $\alpha_{1}=0.7, \alpha_{2}=0.7$ in (18) respectively.

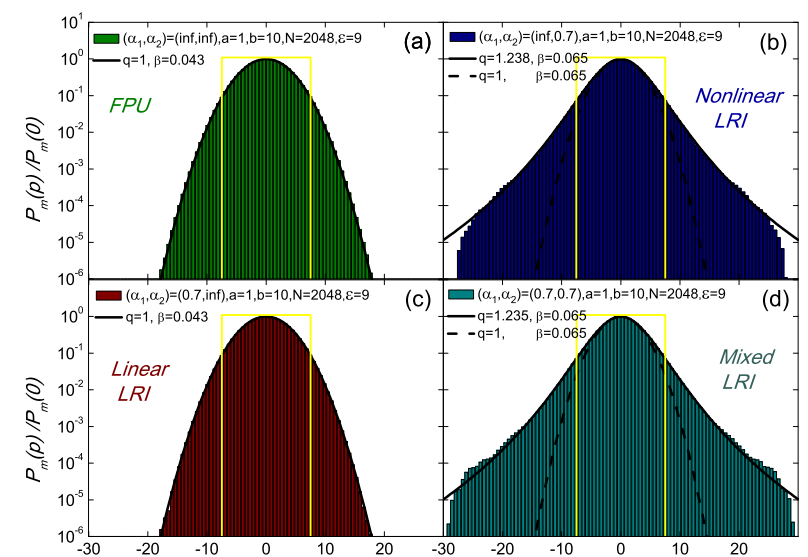

FIG. 12. (color online) The momentum distributions for $N=2048$ particles for the system (16). The upper panels show the cases: $\alpha_{1} \rightarrow \infty, \alpha_{2} \rightarrow \infty$, i.e. FPU (left) and $\alpha_{1} \rightarrow \infty, \alpha_{2}=0.7$ (right). Lower panels show: $\alpha_{1}=0.7, \alpha_{2} \rightarrow \infty$ (left) and $\alpha_{1}=\alpha_{2}=0.7$ (right). The yellow lines correspond to the uniform distribution, from which the momenta where randomly extracted (after [13]).

\subsection{The effect of LRI in 1D lattices with} on-site potential

More recently, I examined in [14] with $\mathrm{H}$. Christodoulidi and L. Drossos, the effect of LRI on 1D Hamiltonian lattices in the presence of onsite potentials that are known to support discrete breathers: the Klein-Gordon (KG) lattice with only quadratic interactions [20] and the GorbachFlach (GF) [21] lattice with purely quartic interactions. Our purpose was to investigate how the localization properties of these lattices are affected by the presence of LRI. The KG lattice, with quadratic and quartic on site potential and linear dispersion terms, is described by the Hamiltonian (LRI with $\alpha=0$ ):

$$
\begin{aligned}
H^{K G-L R I}(p, x) & =\sum_{n}\left\{\frac{1}{2} p_{n}^{2}+\frac{1}{2} x_{n}^{2}+\frac{1}{4} x_{n}^{4}\right. \\
+ & \left.\frac{1}{2(N-1)} \sum_{m}\left(x_{m}-x_{n}\right)^{2}\right\}
\end{aligned}
$$

while the GF model consists of $N$ coupled oscillators with the same KG on-site potential, but with only quartic interactions (LRI with $\alpha=$ $0)$,

$$
\begin{array}{r}
H^{G F-L R I}(p, x)=\sum_{n}\left\{\frac{1}{2} p_{n}^{2}+\frac{1}{2} x_{n}^{2}+\frac{1}{4} x_{n}^{4}\right. \\
\left.+\frac{1}{2(N-1)} \sum_{m}\left(x_{m}-x_{n}\right)^{4}\right\} .
\end{array}
$$

Observe that here we have taken interactions to be of the longest possible range, since the exponent $\alpha$ in the denominator of the double sums (see e.g. (16) or (18) is set to zero, with all interparticle forces having equal strength.

An interesting result is discovered in these models regarding the behavior of the maximal Lyapunov exponent $\lambda$ as the number of particles $N$ grows to larger and larger values. When we compare its behavior in the short and long-range KG and GF models, we find a striking difference between short and long interactions. As Fig. 13 clearly shows, LRI has a dramatic effect on the chaotic properties of both systems: While, under short-range interactions, $\lambda$ tends to saturate to a positive value, under LRI $\lambda$ decreases towards zero, thus indicating that both systems become less and less chaotic as $N$ increases.

Finally, under LRI, momentum distributions of KG and GF are clearly non-Gaussian, which suggests the presence of weak chaos in these systems as $N$ grows indefinitely. However, the associated pdfs we obtain are not exactly qGaussian either [14]. Thus, 1D lattices with onsite potentials need to be studied further, under LRI, regarding their statistical properties in the thermodynamic limit.

\section{Supratransmission and long range interactions}

4.1. Supratransmission and LRI in the absence of on-site potentials

Supratransmission is a phenomenon that has been extensively studied to date in a wide variety of physical systems. Since its discovery in a 1-D Hamiltonian chain consisting of nonlinear oscillators [23], the process of 


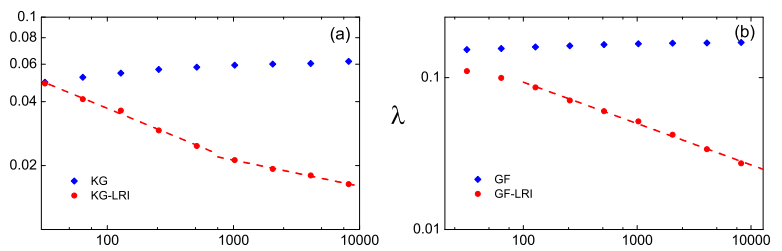

FIG. 13. (color online) The maximal Lyapunov exponent for an increasing number of particles $N$. In the LRI cases, the maximal Lyapunov exponent decreases as $\lambda \propto N^{-0.12}$ in the $\mathrm{KG}$ model, while $\lambda \propto N^{-0.27}$ in the GF (here $\mathrm{KG}, \mathrm{GF}$ refer to nearest neighbor interactions). (a) KG (diamonds) and $\mathrm{KG}$-LRI (circles). (b) GF (diamonds) and GFLRI (circles) (after[14])

nonlinear supratransmission has been found in many other systems, including discrete arrays consisting of anharmonic oscillators [24], linear arrays of Josephson junctions attached through superconducting wires [25] and a nearestneighbor $\beta$-FPU chain [27], to mention some examples. Recently, J. C. E. Macias Diaz and I studied the phenomenon of supratransmission on an $N$-particle 1D $\beta$-FPU lattice, under different ranges of interactions [26]. More specifically, we set the first particle in sinusoidal motion at a frequency $\Omega$ that lies in the "forbidden gap" (i.e. outside the spectrum of normal modes) of the linear nearest-neighbor FPU chain and used suitable computational techniques to extend earlier results of supratransmission to FPU chains with longer ranges of particle interactions than simply nearest neighbors. In other words, as I explained in Section 3, we considered interactions between all particles and introduced scaling exponents $0<\alpha_{1}, \alpha_{2}<\infty$ to quantify the extent of LRI in the model as follows:

$$
\begin{aligned}
\mathcal{H}=\frac{1}{2} \sum_{n=1}^{N} p_{n}^{2} & +\frac{a}{2 M_{1}} \sum_{n=0}^{N} \sum_{m=n+1}^{N+1} \frac{\left(x_{m}-x_{n}\right)^{2}}{(m-n)^{\alpha_{1}}} \\
& +\frac{b}{4 M_{2}} \sum_{n=0}^{N} \sum_{m=n+1}^{N+1} \frac{\left(x_{m}-x_{n}\right)^{4}}{(m-n)^{\alpha_{2}}},
\end{aligned}
$$

where the scaling factors

$$
M_{i}=\frac{1}{N+1} \sum_{n=0}^{N} \sum_{m=n+1}^{N+1} \frac{1}{(m-n)^{\alpha_{i}}}, \quad \forall i \in\{1,2\} .
$$

are introduced to make all three parts of the Hamiltonian extensive, i.e. proportional to $N$ [26]. Our first discovery was that, for a very wide range of $\alpha_{1,2}$ values, supratransmission occurs at similar amplitude thresholds as in the nearest neighbor case. However, as the interaction range becomes longer (and $\alpha_{1,2}$ values decrease towards zero), the threshold amplitude for supratransmission rises to higher and higher values!.

To see exactly what happens we first computed supratransmission kinetic energy thresholds as a function of the driving amplitude, fixing the value of $\alpha_{1}$ and varying the value of $\alpha_{2}$. Next, we repeated the same experiment fixing the value of $\alpha_{2}$ in each frame and varying the value of $\alpha_{1}$. Remarkably, as long as the $\alpha_{1}$ increases and the quadratic interaction become short-range, supratrasmission occurs at higher and higher thresholds, which reach (for $0<\alpha_{2}<1$ ) the value 10 and above (see Fig. 14).

Thus, our main results in this subsection can be summarized as follows:

- Nonlinear supratransmission exists in the system (22) for every pair $\left(\alpha_{1}, \alpha_{2}\right) \in$ $[0, \infty] \times[0, \infty]$.

- The critical amplitude at which supratransmission occurs depends continuously on $\alpha_{1}$ and $\alpha_{2}$, and increases monotonously as $\alpha_{1}$ and $\alpha_{2}$ decrease. This was true for all $\Omega$ we tried within the forbidden band-gap $[2, \infty)$.

- Moreover, when $\alpha_{1}, \alpha_{2} \geq 5$ the supratransmission threshold coincides with that of the $\beta$-FPU chain with nearest-neighbor interactions.

- Interestingly, we found that as long as $\alpha_{2}=0$, i.e. in the case of maximal LRI in the quartic part of the potential, the supratransmission threshold amplitude reaches a maximum value of $A_{s}\left(\alpha_{1}, 0\right) \approx$ 7.6 independent of the value of $\alpha_{1} \in[0, \infty)$.

- In fact, when we considered stronger long range values of $0<\alpha_{2}<1$ the threshold amplitude increased further to a value about 10.3, as $\alpha_{1} \longrightarrow \infty$ (see Fig. 14). 

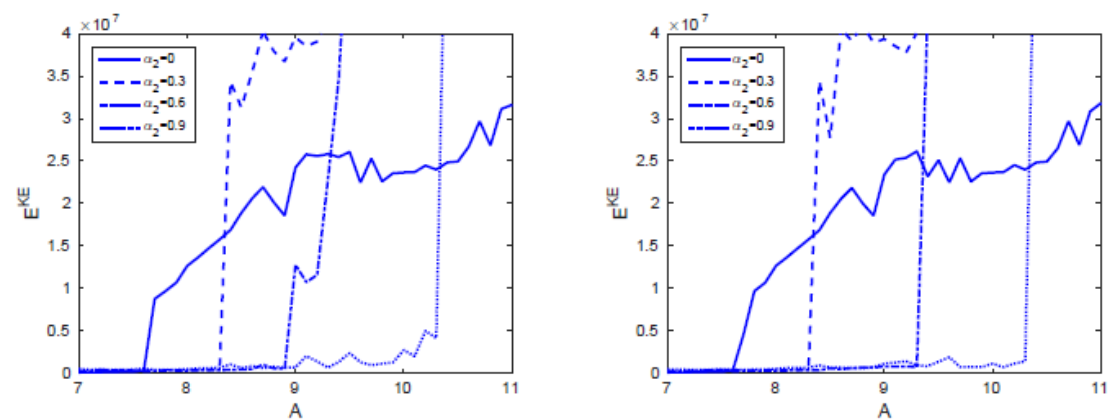

FIG. 14. (color online) Graphs of the total kinetic energy versus driving amplitude A of the system (22) with $\Omega=3.5$. The results were obtained using (left) $\alpha_{1}=10$ and (right) $\alpha_{1}=\infty$. The graphs correspond to $\alpha_{2}=0$ (solid), $\alpha_{2}=0.3$ (dashed), $\alpha_{2}=0.6$ (dash-dotted) and $\alpha_{2}=0.9$ (dotted) (after [26]).

(a)

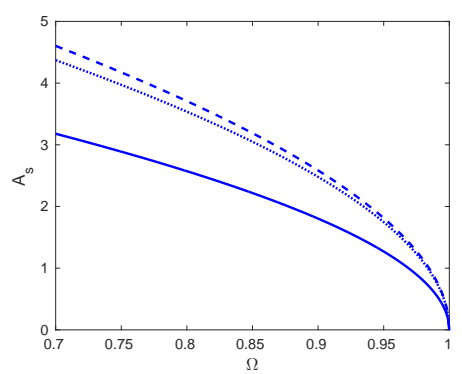

(b)

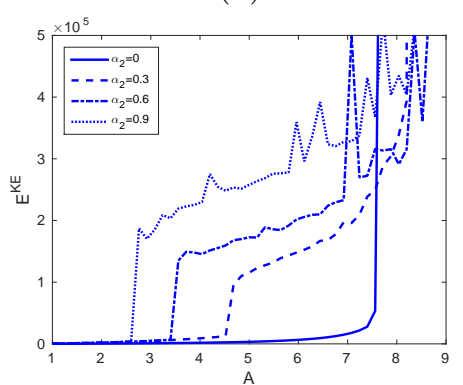

FIG. 15. (color online) Graphs of the approximate critical amplitude at which supratransmission is triggered versus $\Omega$, for (a) $\alpha=2$ and (b) $\alpha=3$. The parameters are as in previous figures. The solid curve is the short range interaction function (28), the dashed lines are the numerical approximations, and the dotted curve is the plot of the empirical formula (28) (after [16]).

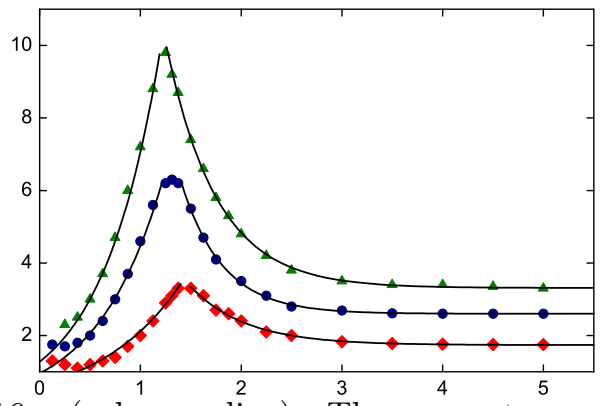

FIG. 16. (color online) The supertransmission threshold $A_{s}$ as a function of $\alpha$ for $\Omega=0.9$ (diamonds), $\Omega=0.8$ (circles) and $\Omega=0.7$ (triangles) (after [16]).

\subsection{Supratransmission and LRI in the} presence of on-site potentials

A suprising result awaited us, however, when we studied recently the effect of LRI on supratransmission thresholds, in the case of 1D Hamiltonians, whose potential includes on-site potentials [16]. Our system is initially at rest at the equilibrium position, and is described by the Hamiltonian

$$
\begin{array}{r}
\mathcal{H}=\sum_{n=1}^{N}\left(\frac{1}{2} p_{n}^{2}+V\left(x_{n}\right)\right) \\
+\frac{1}{p M} \sum_{n=0}^{N} \sum_{m=n+1}^{N+1} \frac{W\left(x_{m}-x_{n}\right)}{(m-n)^{\alpha}} .
\end{array}
$$

Here, the number $\alpha$ governs the "length" of the particle interactions, with $\alpha=\infty$ denoting the shortest possible range of nearest neighbor interactions. Thus, we shall say that the system possesses long range interactions if $\alpha<\infty$, with the strongest LRI corresponding to $0 \leq \alpha \leq 1$. The scaling factor

$$
M=\frac{1}{N+1} \sum_{n=0}^{N} \sum_{m=n+1}^{N+1} \frac{1}{(m-n)^{\alpha}}
$$

is used to make the last term at the right-hand side of (24) extensive, that is, proportional to 
$N$. As a consequence, the associated equations of motion of our model are given by

$$
\ddot{x}_{n}=\frac{1}{p M} \sum_{\substack{m=1 \\ m \neq n}}^{N} \frac{W^{\prime}\left(x_{m}-x_{n}\right)}{|m-n|^{\alpha}}-V^{\prime}\left(x_{n}\right),
$$

for each $n \in\{1, \ldots, N\}$. It is worth noting that (26) extends various well known models of mathematical physics to account for LRI. In [16], we treated the classical sine-Gordon chain when $V(u)=1-\cos (u)$ and checked that the solutions and phenomena observed with (26) are very similar with what one finds for the potential $V(u)=\frac{1}{2 !} u^{2}-\frac{1}{4 !} u^{4}+\frac{1}{6 !} u^{6}$, and the double sineGordon chains when $V(u)=\frac{1}{2}-\frac{1}{6}[2 \cos u+$ $\cos (2 u)]$.

We will, therefore consider a system of unit mass particles whose positions satisfy the initialboundary-value problem

$$
\begin{aligned}
& \ddot{x}_{n}=-\gamma_{n} \dot{x}_{n}+\frac{1}{p M} \sum_{\substack{m=1 \\
m \neq n}}^{N} \frac{W^{\prime}\left(x_{m}-x_{n}\right)}{|m-n|^{\alpha}}-V^{\prime}\left(x_{n}\right), \\
& \text { such that }\left\{\begin{array}{l}
x_{0}(t)=A \sin (\Omega t), \quad t \geq 0, \\
x_{N+1}(t)=x_{N}(t), \quad t \geq 0, \\
x_{n}(0)=\dot{x}_{n}(0)=0, \quad 1 \leq n \leq N+1
\end{array}\right.
\end{aligned}
$$

where $1 \leq n \leq N$ and $A, \Omega$ are positive. Clearly, (27) represents a system of particles which is initially at rest, and is perturbed harmonically at the left end, with the presence of a free boundary at the last particle on the right. Moreover, to model a long chain we let $N$ be relatively large, and use damping coefficients, $\gamma_{n} \in \mathbb{R}^{+} \cup\{0\}$ to simulate an absorbing boundary at the right.

We now assume that the frequency $\Omega$ belongs to a "forbidden" band-gap, here $0<$ $\Omega<1$, that lies (together with its harmonics) outside the phonon spectrum of the system. As the driving amplitude is increased, a critical value $A_{s}$ is reached above which the system suddenly absorbs great amounts of energy from the oscillating boundary. The number $A_{s}$ is called the supratransmission threshold, and in the case of Klein-Gordon type systems an analytical approximation of its value at $\alpha=\infty, p=2$ and $\gamma_{n}=0$ for each $n \in\{1, \ldots, N\}$, is known [23]

$$
A_{s}(\Omega)=4 \arctan \left[\frac{c}{\Omega} \operatorname{arccosh}\left(1+\frac{1-\Omega^{2}}{2 c^{2}}\right)\right]
$$

for each $\Omega \in(0,1)$, and $c=a / \sqrt{M}$. It is worth noting that this approximation is valid for relatively large values of $c$, and for values of $\Omega<1$ which are close to 1 .

Now let us set $\Omega=0.7$ and study the behavior of the solution of (27) versus $n$ and $t$, for various values of $\alpha$ and $A$ [16]. In the strong LRI case, $\alpha=0,0.5,1$, we found clear evidence of supratransmission at threshold amplitudes $A_{s}$, which decrease as $\alpha$ decreases in this range! In fact, this holds for all other values of $\Omega>0.7$. This aspect of our results suggests a complex relationship between the driving frequency and the critical amplitude at which supratransmission is triggered in the regime of strong LRI. In Figure 16 we have plotted the supratransmission threshold $A_{s}(\alpha)$ for the full range of $\alpha$ LRI values for three values of the driving frequency: $\Omega=0.9$ (diamonds), $\Omega=0.8$ (circles) and $\Omega=0.7$ (triangles). We suggest the following explanation for this sudden decrease of $A_{s}$ as $\alpha$ goes to zero: As was observed in our recent study of Klein-Gordon type systems [14], in the limit of $\alpha \rightarrow 0$ the phonon band shrinks to a line and all particles become so strongly correlated that energy transmission can occur for very small driving amplitudes, as shown in Figure 16. Now, what happens exactly in that limit is not clear, since the very nature of supratransmission becomes doubtful in the strong LRI regime $\alpha<$ 0.5 .

\section{Future outlook}

As is well-known, the field of modeling complex materials has been expanding rapidly in recent years, with the aim of understanding the dynamical response of metallic structures used in mechanical engineering applications. In this regard, I have been studying recently with Dr. Kostas Kaloudis and Dr. Thomas Oikonomou [34]1-D Hamiltonian lattices of particles interacting via 1) graphene type interactions [28-30], 2) Hollomon's power-law of materials exhibiting "work hardening" [31-33]. Earlier studies have focused on the dynamics of single oscillators governed by suitable nonanalytic potentials describing the motion in the above two cases. Our main aim in this direction is to extend these studies to 1-D Hamiltonian 
lattices and perform a local and global stability analysis of simple periodic oscillations to identify energy and parameter regions associated with globally stable motion as well as regimes of strong and weak chaos. Thus, we have begun to carry out an analysis similar to what was described in the first four sections of this paper, to the following 1-D lattices:

$H=\sum_{j=1}^{n} \frac{1}{2} \dot{x}_{j}^{2}+\sum_{j=0}^{n} \frac{K}{2}\left(x_{j+1}-x_{j}\right)^{2}-\frac{D}{3}\left|x_{j+1}-x_{j}\right|^{3}$

as a graphene 1-D model and

$$
\begin{array}{r}
H=\frac{1}{2} \sum_{j=1}^{N} m_{j} \dot{x}_{j}^{2}+\frac{k}{2} \sum_{j=0}^{N}\left(x_{j+1}-x_{j}\right)^{2} \\
+\frac{\lambda}{q+1} \sum_{j=0}^{N}\left|x_{j+1}-x_{j}\right|^{q+1}
\end{array}
$$

as a Hollomon's law model. Here $K$ is an elastic constant and $D>0$ represents material stiffness, $\lambda>0$ and the exponent $0<q<1$ captures the relevant fractional nonlinearities of the model. For $q=1$ the former equation recovers the Hamiltonian of a system of coupled harmonic oscillators, while for $q=3$ it reduces to the Hamiltonian of the $\beta$ - FPU lattice.

Preliminary results demonstrate that the above systems possess stability properties that are strikingly different than those of FPU 1D Hamiltonian lattices [34]. In particular, the graphene type Hamiltonian (29) possesses SPO1 and SPO2 orbits that first destabilize along $E_{c} / N$ vs. $N$ curves that go to zero as $N$ grows with quite different power laws than in the FPU case.
On the other hand, the behavior of the Hollomon Hamiltonian (30) is even more remarkable: Its SPO1 and SPO2 solutions are unstable at low energies and first stabilize along curves that grow with positive powers of $N$ as the number of particles increases!

Both models have so far been studied under fixed boundary conditions and nearestneighbor interactions [34]. However, motivated by the approaches described in this review, we also plan, in the near future, to analyze their dynamics under LRI and investigate whether they also exhibit the phenomenon of nonlinear Supratransmission.

\section{Acknowledgements}

In this paper, I review a number of results that my co-workers and I have obtained over many years. Many of them are former Ph.D. students of mine that now hold positions at international academic and research institutions. Of the more senior collaborators that crucially participated in the research described here, I would like to thank Professor Constantino Tsallis, and Professor Jorge Macias Diaz. I am grateful to Professor Angela de Sanctis for organizing the 6th Ph.D. Conference- Summer School in July of 2019 at Pescara, and I wish to thank Professor George Krylov and the Journal of Nonlinear Phenomena in Complex Systems for their initiative to publish some of the papers presented during that event. Finally, I would like to acknowledge my Nazarbayev University ORAU grant, 2017-2020, under whose auspices many of the results of Sections 3 and 4 of this paper have been obtained.

\section{References}

[1] V.I. Arnold. Mathematical Methods of Classical Mechanics. (Springer, New York, 1989).

[2] A.J. Lichtenberg, M.A. Lieberman. Regular and Chaotic Dynamics. Second edition. (Springer, New York, 1992).

[3] S. Wiggins. Applied Nonlinear Dynamical Systems and Chaos. (Springer, New York, 1990).

[4] T. Bountis, H. Skokos. Complex Hamiltonian
Dynamics. Vol. 10. (Springer Science \& Business Media, Berlin, 2012).

[5] A.M. Lyapunov. The General Problem of the Stability of Motion. (Taylor and Francis, London, 1992).

[6] G.P. Berman, F.M. Izrailev. The Fermi-PastaUlam problem: Fifty Years of Progress. Chaos, 15, 015104 (2005). 
[7] Ch. Antonopoulos, T. Bountis. Stability of Simple Periodic Orbits and Chaos in a FermiPasta-Ulam Lattice. Phys. Rev. E. 73, 56-206 (2006).

[8] Ch. Antonopoulos, T.C. Bountis, Ch. Skokos. Chaotic Dynamics of N-degree of Freedom Hamiltonian Systems. Int. J. Bifurcat. Chaos, 16, 1777-1793 (2006).

[9] Ch. Skokos, T. Bountis, C. Antonopoulos. Geometrical Properties of Local Dynamics in Hamiltonian Systems: The Generalized Alignment (GALI) Method. Physica D. 231, 30 (2007).

[10] Ch. Skokos, T. Bountis, C. Antonopoulos. Detecting Chaos, Determining the Dimensions of Tori and Predicting Slow Diffusion in Fermi-Pasta-Ulam Lattices by the Generalized Alignment Method. European Physics Journal, Special Topics. 165, 5 - 14 (2008).

[11] H. Christodoulidi, C. Tsallis, T. Bountis. Fermi-Pasta-Ulam Model with Long Range Interactions: Dynamics and Thermostatistics. European Physics Journal Lett. 108, 40006 (2014).

[12] C. Anteneodo, C. Tsallis. Breakdown of Exponential Sensitivity to Initial Conditions: Role of the Range of Interactions. Phys. Rev. Lett. 80, 5313 (1998).

[13] H. Christodoulidi, T. Bountis, C. Tsallis, L. Drossos. Chaotic Behavior of the Fermi-PastaUlam Model with Different Ranges of Particle interactions. J. Stat. Mech. 12 (12), 123206 (2016).

[14] H. Christodoulidi, A. Bountis, L. Drossos. The Effect of Long-range Interactions on the Dynamics and Statistics of 1D Hamiltonian Lattices with On-Site Potential. EPJST. 227(5,6) 563 (2018).

[15] J.C. Macias Diaz, A. Bountis. On the Transmission of Energy in $\beta$-Fermi-PastaUlam Chains with Different Ranges of Particle Interactions. CNSNS. 63, 307-321 (2018).

[16] J. E. Macias-Diaz, A. Bountis, H. Christodoulidi. Energy Transmission in Hamiltonian Systems with Globally Interacting Particles and On-Site Potentials. Mathematics in Engineering. 1(2), 343-358 (2019).

[17] G. Benettin, L. Galgani, A. Giorgilli, J.-M. Strelcyn. Lyapunov Characteristic Exponents for Smooth Dynamical Systems and for Hamiltonian Systems; a Method for Computing All of Them. Meccanica 15, 9-30 (1980).

[18] C. Tsallis. Introduction to Nonextensive Statistical Mechanics: Approaching a Complex World. (Springer, New York, 2009).

[19] Ch. Antonopoulos, T. Bountis, V. Basios. QuasiStationary Chaotic States of Multidimensional
Hamiltonian Systems. Physica A. 390, 32903307 (2011).

[20] R.S. MacKay, S. Aubry. Proof of Existence of Breathers for Time-Reversible or Hamiltonian Networks of Weakly Coupled Oscillators. Nonlinearity. 7, 1623 (1994).

[21] A.V. Gorbach, S. Flach. Compactlike Discrete Breathers in Systems with Nonlinear and Nonlocal Dispersive Terms. Phys. Rev. E. 72, 056607 (2005).

[22] Ch. Skokos. The Lyapunov Characteristic Exponents and Their Computation. Lect. Notes Phys. 790, 63-135 (2010).

[23] F. Geniet, J. Leon. Energy Transmission in the Forbidden Band Gap of a Nonlinear Chain. Phys. Rev. Lett. 89(13), 134102 (2002).

[24] R. Khomeriki, S. Lepri, S. Ruffo. Nonlinear Supratransmission and Bistability in the FermiPasta-Ulam model. Phys. Rev. 70(6), 066626 (2004).

[25] D. Chevriaux, R. Khomeriki, J. Leon. Theory of a Josephson Junction Parallel Array Detector Sensitive to Very Weak Signals, Phys. Rev. B. 73(21), 214516 (2006) .

[26] J.C.E. Macias Diaz, A. Bountis. On the Transmission of Energy in $\beta$-Fermi-PastaUlam Chains with Different Ranges of Particle Interactions. CNSNS. 63, 307-321 (2018).

[27] J.C.E. Macias Diaz, Numerical Simulation of the Nonlinear Dynamics of Harmonically Driven Riesz-Fractional Extensions of the Fermi-PastaUlam Chains. CNSNS. 55, 248 (2018).

[28] E. Cadelano, P.L. Palla, S. Giordano, L. Colombo. Nonlinear Elasticity of Monolayer Graphene. Phys. Rev. Lett. 102(23), 235502 (2009).

[29] H. Hazim, D. Wei, M. Elgindi, Y. Souklassian. A Lumped-Parameter Model for Nonlinear Waves in Graphene. World J. Eng. Tech. 3, 57 -69 (2015).

[30] D. Wei, S. Kadyrov, Z. Kazbek. Periodic Solutions of a Graphene Based Model in a MicroElectro-Mechanical Pull-in Device. Appl. Comp. Mech. 11(1), 1-10 (2017).

[31] J.H. Hollomon. Tensile deformation. Transactions of the Metallurgical Society of AIME. 162, 268-290 (1945).

[32] D. Wei, Yu Liu. Some generalized trigonometric sine functions and their applications. Applied Mathematical Sciences 6(122), 6053-6068 (2012).

[33] D. Wei, A. Sarria, M. Elgindi. Critical Buckling Loads of the Perfect Hollomon's Power-law Columns. Mech. Res. Comm. 47, 69-76 (2013).

[34] A. Bountis, K. Kaloudis, T. Oikonomou. Stability and chaos in 1-D Hamiltonian lattices with non-analytic potentials. In preparation (2019). 